\title{
MEDIÇÕES ESPECTROFOTOMÉTRICAS DA OXIDAÇÃO NO DOSÍMETRO QUÍMICO FRICKE XYLENOL GEL (FXG): AVALIAÇÃO DAS DISPOSIÇÕES DAS AMOSTRAS PÓS- IRRADIAÇÕES
}

\section{N. OLIVEIRA, A. ALMEIDA e L. V. E. CALDAS}

Instituto Federal de Educação, Ciência e Tecnologia de Goiás - IFG/Campus Inhumas Faculdade de Filosofia, Ciências e Letras de Ribeirão Preto - FFCLRP/Universidade de São Paulo-USP Instituto de Pesquisas Energéticas e Nucleares - IPEN/CNEN lucas@ifg.edu.br

Artigo submetido em dezembro/2013 e aceito em julho/2016

DOI: $10.15628 /$ holos.2016.1890

\section{RESUMO}

O método espectrofotométrico foi usado para determinação da oxidação em gel dosimétrico químico. Este baseia-se sobre a reação de $\mathrm{Fe}^{2+}$ para $\mathrm{Fe}^{3+}$ o qual a leitura aumenta de acordo com o sinal de absorbância em $585 \mathrm{~nm}$. O dosímetro químico foi irradiação e mantido sobre as posições horizontais e verticais com e sem tampa para analises de oxidação devido à radiação ou contato com oxigênio. O método foi aplicado com sucesso para a determinação de oxidação em Fricke Xylenol Gel (FXG), e os resultados estão de acordo com a posição melhor acomodação deste dosímetro gel química.

PALAVRAS-CHAVE: Dosímetro Químico Gel, Oxidação, Espectrofotometria.

\section{SPECTROPHOTOMETRIC MEASUREMENTS OF THE OXIDATION IN CHEMICAL DOSIMETER FRICKE XYLENOL GEL (FXG): EVALUATION OF THE PROVISIONS OF THE POST-IRRADIATION SAMPLES}

\begin{abstract}
The spectrophotometric method was used for oxidation determination in chemical gel dosimeter. The method based on the reaction of the $\mathrm{Fe}^{2+}$ to $\mathrm{Fe}^{3+}$ lead to increases in the magnitude of the absorbance signal monitored at $585 \mathrm{~nm}$. The chemical dosimeter was irradiated and maintained in the horizontal and vertical positions with
\end{abstract}

and without cover for the analysis of oxidation due to radiation or contact with oxygen. The method was successfully applied for oxidation determination in Fricke Xylenol Gel (FXG), and the results agree with the better accommodation position of this chemical gel dosimeter.

KEYWORDS: Chemical Gel Dosimeter; Oxidation; Spectrophotometry 


\section{INTRODUÇÃO}

Em Física das Radiações, conceitos básicos de interação da radiação com a matéria são necessários, fundamentalmente no tocante de energia sendo absorvida. Segundo Attix (1993, p.5), têm-se a seguinte definição:

Radiações ionizantes são geralmente caracterizadas pela sua habilidade de excitar ou ionizar átomos da matéria com os quais tenham interações. Conseqüentemente, a determinação da energia depositada na matéria por essas radiações são freqüentemente chamadas de dosimetria.

Para a quantificação da dose depositada pela radiação no meio, tem-se a dose absorvida, definida por Johns (1983, p.10):

Dose absorvida é definida como a energia depositada pela radiação ionizante por unidade de massa no material e é expressa em J/kg. Esta é uma importante característica em ciência radiológica que é uma unidade especial no Sistema Internacional (SI), o gray (Gy) que tem sido criado para representar $1 \mathrm{~J} / \mathrm{kg}$.

Em Física Médica, medidas de dose absorvida são realizadas através de dosímetros como: câmara de ionização, filmes, termoluminescentes, químicos os quais possuem uma resposta linear com a dose absorvida em seu volume sensível (ATTIX, 1993; JOHNS, 1983; KHAN, 1993). Particularmente, os dosímetros químicos Fricke, funcionam a partir do princípio de oxidação dos íons ferrosos $\mathrm{Fe}^{2+}$ em íons férricos $\mathrm{Fe}^{3+}$, esta, induzida por radiação ionizante ou mediante exposição da solução ao oxigênio. A busca de novas soluções dosimétricas é de interesse na área de dosimetria das radiações, e no ano de 2000 houve a adição do alaranjado de xylenol (um indicador de íons férricos) e da gelatina 300 Bloom à solução Fricke padrão criando-se o Fricke Xilenol Gel (FXG) (BERO, 1999; BERO, 2000; BERO 2001). Neste gel dosímetrico, pode-se determinar de maneira indireta a quantidade produzida de íons férricos e ferrosos na solução, empregando a técnica de espectrofotometria, a variação da absorbância associada à incidência de radiação, geralmente, acarreta uma mudança de cor na região do espectro eletromagnético visível, que varia de acordo com a dose absorvida do dosímetro. O FXG apresenta densidade de 1,050 $\mathrm{g} / \mathrm{cm}^{3}$ e número atômico efetivo de 7,75 , estes valores são próximos aos do tecido mole, fato que credencia o FXG para medidas em radioterapia, além disto, possui linearidade com a dose absorvida entre 0,5 e 30 Gy, com pico de absorbância máxima em 585 nm (ALVA-SÁNCHEZ, 2014; OLIVEIRA, 2007; OLIVEIRA 2009a; OLIVEIRA 2009b).

O desenvolvimento da dosímetria química Fricke, está associado aos avanços em seu sistema de leitura de baixo custo (PIRANI, 2013; SAMPAIO, 2013a; SAMPAIO 2013b), desenvolvimento de formulações mais sensíveis (PIRANI, 2009), coeficiente de difusão (DAVIES, 2008; DAVIES, 2010; DAVIES, 2013), cálculos fundamentais em dosimetria das radiações (CALCINA, 2007), estas sendo aplicadas em serviços de radioterapia. O leitor pode encontrar na literatura especializada como: Medical Physics, Physics Medicine and Biology, Radiation Measurements, Radiation Physics and Chemistry, Applied Radiation and Isotopes, Surface and Coatings Technology e Medical Dosimetry, a interdisciplinaridade entre as áreas de Química e Física Médica, as quais possuem os avanços da dosimetria química que são aplicados à área médica, principalmente no controle de qualidade dos equipamentos utilizados em tratamentos de cânceres. 
A oxidação natural ou induzida por radiação em dosímetros químicos deve ser pesquisada, pois, após a irradiação ou mesmo espontaneamente, a formação de íons $\mathrm{Fe}^{+3}$ na solução química pode acarretar medições incorretas e consequentemente no momento da leitura esta pode apresentar valores subestimados na determinação da dose absorvida. O controle da oxidação, ou seja, determinar a resposta espaço-temporal do FXG, é de fundamental importância para a área de dosimetria química. Isto devido a sua utilização em medições relacionadas num serviço de radioterapia, as quais devem ser corrigidas pelo coeficiente de difusão encontrado para o dosimetro. Essas correções poderiam ser feitas a qualquer tempo, retornando aos valores originais, ou seja, imediatamente pós-irradiação. O sinal do FXG ( $\mathrm{Fe}^{+3}$-Xylenol) difunde modificando as leituras das absorbâncias, especialmente aquelas realizadas após $1 \mathrm{~h}$ da incidência da radiação na amostra (OLIVEIRA, 2009a). Outro detalhe, para o coeficiente de difusão, são seus valores associados para dosimetros géis, os quais, estão no interval de 0,3 até $2 \mathrm{~mm}^{2} / \mathrm{h}$ (KRON, 1997). Estudos da oxidação com FXG podem sem encontrados, seja, utilizando a técnica de fotoacústica (CALDEIRA, 2007a, CALDEIRA, 2007b), a qual não é comumente utilizada em laboratórios de Física Médica; ou, usando a técnica da espectrofotometria (OLIVEIRA, 2009a, DAVIES, 2008), mas em ambos os casos estas pesquisas não levam em consideração as possíveis posições em que o dosímetro possa ser acomodado durante seu armazenamento e leitura.

Portanto, o objetivo deste trabalho é a determinação da oxidação no dosímetro químico FXG, medida espectrofotometicamente, provocada pela exposição da amostra a irradiação ou ao ambiente (natural). Pela primeira vez, a condição de armazenamento e leitura dos dosímetros foram analisadas em duas situações pós-irradiações: i) cubetas nas posições verticais e horizontais de acomodação e ii) cubetas com e sem tampa na mesma configuração do item (i).

\section{METODOLOGIA}

\subsection{Preparo do dosímetro químico}

$\mathrm{Na}$ preparação do FXG, foi utilizada a gelatina 300 Bloom, água Milli-Q, à pressão e temperatura ambientes. O primeiro passo, no preparo do FXG, é hidratar a gelatina em um béquer com $75 \%$ da água a ser utilizada na solução (béquer 1). Após 10 minutos da adição da água no béquer, a gelatina hidratada deve ser aquecida; até atingir $40{ }^{\circ} \mathrm{C}$, o aquecimento deve ser desligado. Posteriormente, esta solução é misturada em um agitador magnético que deve permanecer ligado durante todo o preparo da solução. Enquanto a solução gelatinosa atinge a temperatura necessária para o preparo da solução final $\left(30{ }^{\circ} \mathrm{C}\right)$, prepara-se a segunda parte da solução dosimétrica (béquer 2), com sulfato ferroso II, alaranjado de xilenol, ácido sulfúrico com os $25 \%$ restantes da água. Assim que a solução atingir $30{ }^{\circ} \mathrm{C}$, deve ser acrescentada à solução do béquer 2 ao do béquer 1 e manter á solução final em agitação até a completa homogeneização. Após o preparo da solução química, esta foi inserida em cubetas padrão de PMMA com dimensões de 1,25 × 1,25 × 4,5 cm³ , e levadas a um refrigerador para formação de gel, numa temperatura de $10{ }^{\circ} \mathrm{C}$. Na Tabela 1, são mostradas as concentrações e informações dos reagentes para o preparo da solução química Fricke (BERO, 2000). 
Tabela 1. Composição química para a solução dosímetrica Fricke Xilenol Gel (BERO, 2000).

\begin{tabular}{c|c|c|c}
\hline Substância & Fórmula molecular & $\begin{array}{c}\text { Peso molecular } \\
\left(\mathrm{g} \cdot \mathrm{mol}^{-1}\right)\end{array}$ & $\begin{array}{c}\text { Concentração } \\
(\mathrm{mM})\end{array}$ \\
\hline Gelatina 300 bloom & $\left(\mathrm{C}_{17} \mathrm{H}_{12} \mathrm{~N}_{5} \mathrm{O}_{6}\right)$ & 402,47 & 124,38 \\
\hline Alaranjado de Xilenol & $\mathrm{C}_{32} \mathrm{H}_{28} \mathrm{~N}_{2} \mathrm{O}_{11} \mathrm{SNa}_{4}$ & 760,58 & 0,1 \\
\hline Sulfato Ferroso & $\mathrm{Fe}\left(\mathrm{NH}_{4}\right)_{2}\left(\mathrm{SO}_{4}\right)_{2} 6 \mathrm{H}_{2} \mathrm{O}$ & 392,47 & 0,5 \\
\hline Ácido Sulfúrico & $\mathrm{H}_{2} \mathrm{SO}_{4}$ & 98,07 & 25 \\
\hline
\end{tabular}

2.2 Irradiação do dosímetro químico As cubetas contendo o gel dosímetrico foram irradiadas com feixes de fótons do acelerador linear VARIAN CLINAC 6/100 pertencente ao Hospital Araújo Jorge (Goiânia-Goiás), com energia de $6 \mathrm{MV}$, sendo a taxa de dose igual a $200 \mathrm{cGy} / \mathrm{min}$ em um campo de $10 \times 10 \mathrm{~cm}^{2}$, dose absorvida de $10 \mathrm{~Gy}$, a uma distância da fonte-superfície (DFS) de $100 \mathrm{~cm}$. O campo foi fechado em uma das partes para que uma parte ficasse com irradiação e a outra bloqueando a irradiação (Figura 1).

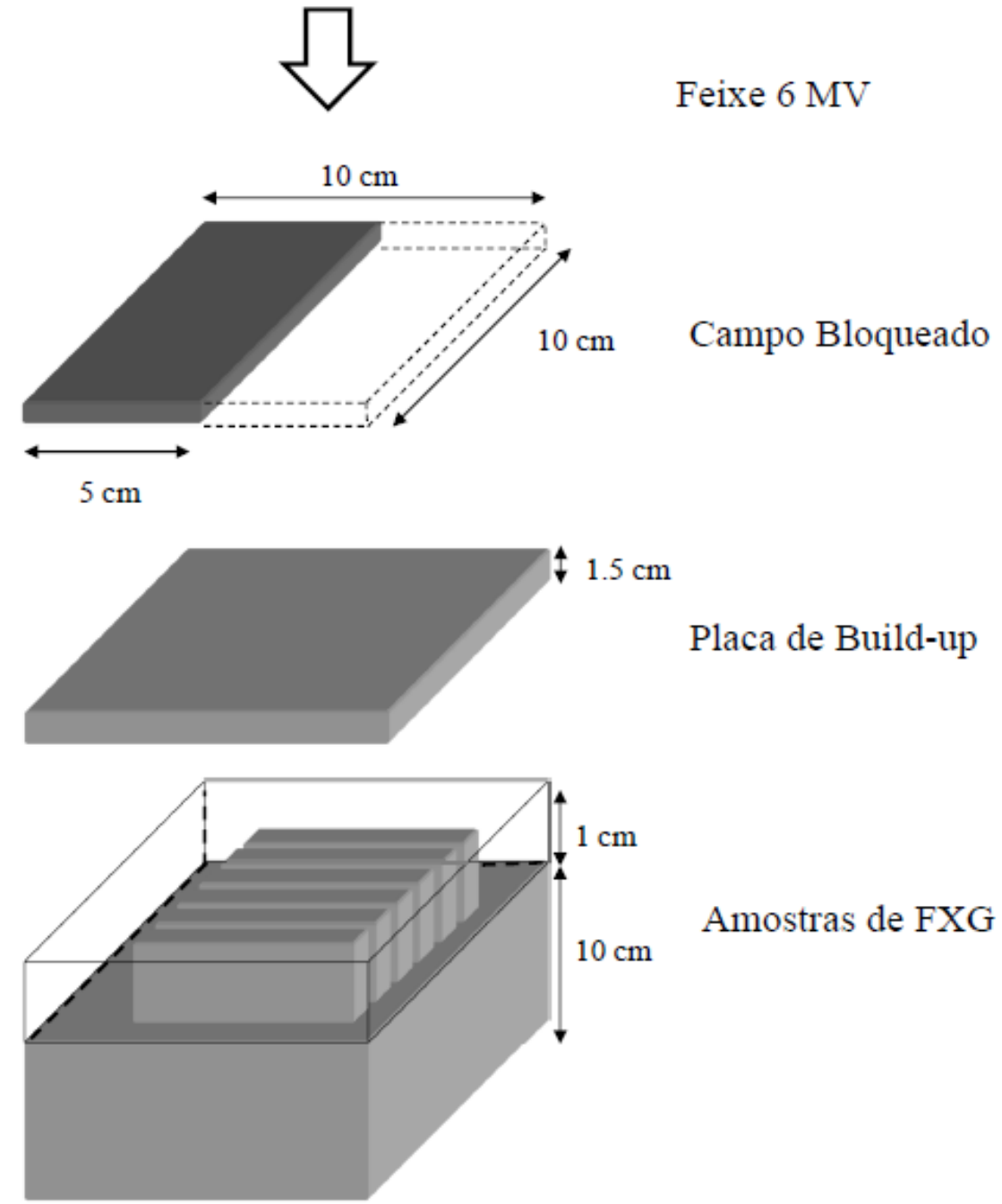

Figura 1. Arranjo experimental com cubetas contendo FXG envolvidas com placas de retroespalhamento (inferior) e build-up (superior), campo blindado em sua metade, sendo irradiado com feixes de 6MV de fótons. 


\subsection{Posicionamento das cubetas contendo o dosímetro químico}

As cubetas, imediatamente após a irradiação, foram posicionadas em duas formas, horizontal e vertical, e associadas a estas posições receberam uma tampa ou não em sua parte superior, conforme Figura 2.

a)

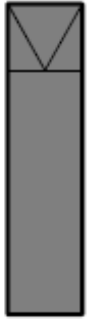

c)

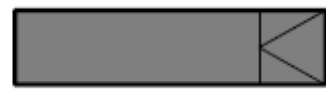

b)

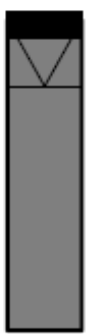

d)

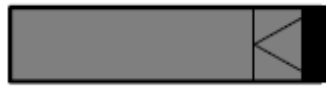

Figura 2. Posições relativas das cubetas contendo o dosímetro químico após irradiação com feixes de fótons. a) e b) cubetas na posição horizontal sem e com tampa respectivamente, c) e d) cubetas na posição vertical sem e com tampa respectivamente.

\subsection{Leitura do dosímetro químico}

No caso de um dosímetro químico Fricke à base de íons $\mathrm{Fe}^{+2}$, quando irradiado, os íons são convertidos em $\mathrm{Fe}^{+3}$, proporcionalmente à quantidade da dose absorvida pela amostra. Dessa maneira, a dose absorvida pode ser inferida pela concentração de $\mathrm{Fe}^{+3}$, por meio da espectrofotometria visível. Essa concentração está relacionada à equação de Lambert-Beer (Equação 1), como:

$$
A=\log \left(\frac{I_{0}}{I}\right)
$$

Em que $A$ é a absorbância, $I$ e $I_{0}$ são as intensidades de luz que atravessam uma amostra irradiada e não irradiada. As leituras realizadas com o espectrofotômetro foram feitas ao longo do comprimento maior da cubeta $(45 \mathrm{~mm}$ ), tendo ao todo 35 leituras. As obtenções das leituras foram tomadas após a irradiação das cubetas nos instantes iguais a: $t_{1}=1 \mathrm{~h}, \mathrm{t}_{2}=5 \mathrm{~h}, \mathrm{t}_{3}=10 \mathrm{~h}, \mathrm{t}_{4}=17 \mathrm{~h}, \mathrm{t}_{5}=$

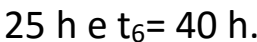

\subsection{Cálculo da oxidação por irradiação e natural}

Os formatos das curvas obtidas com as leituras do dosímetro químico FXG deveriam ser do tipo função degrau, ou seja, um patamar e em seguida uma queda brusca de valores. Este formato é adequado para funções matemáticas que não possuem um agente externo atuando; neste caso especificamente, a penumbra que surge através da radiação ionizante que ao incidir sobre o campo blindado faz com que parte dessa radiação seja espalhada e consequentemente lida no espectrofotômetro. Portanto, a curva que se assemelha às leituras obtidas são da forma sigmoidal, as quais foram ajustadas aos perfis obtidos experimentalmente com o dosímetro químico. 


\section{RESULTADOS E DISCUSSÃO}

A obtenção de resultados da oxidação em géis químicos, dos íons $\mathrm{Fe}^{+2} \mathrm{em} \mathrm{Fe}^{+3}$, que contém os reagentes gelatina 300 Bloom e alaranjado de xylenol (XO) em sua solução, utilizando o método espectrofotométrico foram obtidos, pois este equipamento é adequado em pesquisas científicas na área de dosimetria química e também por ter fácil manuseio e disponibilidade em laboratórios de química.

Na Figura 3 são mostrados os dados experimentais do FXG, a curva sigmoidal ajustada e o cálculo da área sobre a curva sigmoidal (integração), que irá fornecer os resultados da oxidação devido à radiação e quando ocorre naturalmente. A atuação da oxidação é vista no ajuste desta curva, ou seja, tanto a parte inferior e superior os valores das absorbâncias se modificam alterando assim a resposta dosimétrica. A parte inferior contém uma quantidade maior de íons $\mathrm{Fe}^{+2}$ do que na parte superior, dos quais ainda não foram oxidados, e estes estão propensos a serem oxidados em $\mathrm{Fe}^{+3}$, pela ação do ambiente (CALDEIRA, 2007a), fazendo com que o sinal seja elevado nessa região.

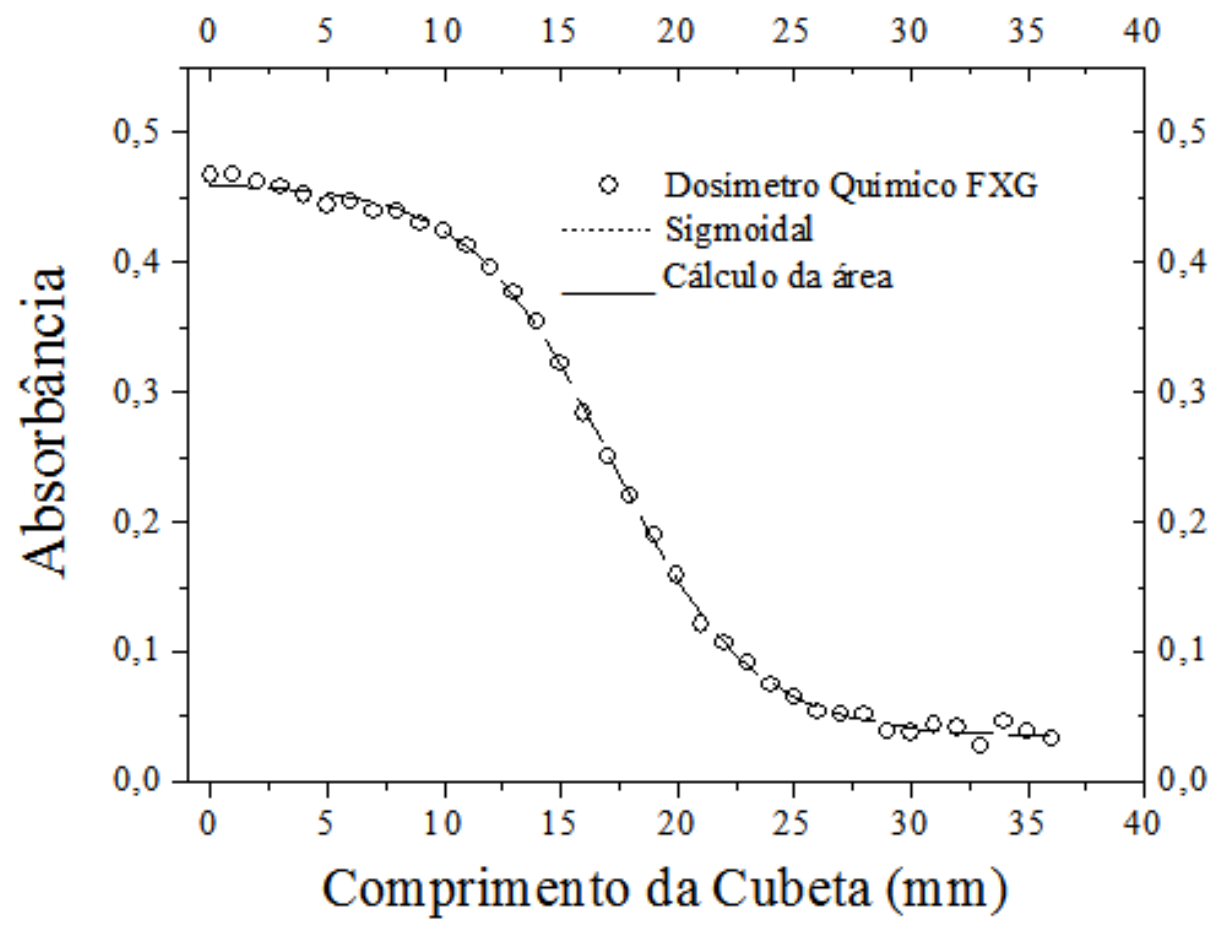

Figura 3. Absorbância proveniente de dados experimentais do dosímetro químico FXG versus comprimento da cubeta, sob a curva sigmoidal pode ser vista a área calculada.

Nas Figuras 4a e 4b são mostradas as áreas sob a curva sigmoidal em função do tempo pósirradiação das amostras do dosímetro químico FXG. Em todos os casos analisados, as amostras sem tampa apresentaram uma oxidação maior em relação ao caso com tampa; este fato pode ser explicado pela maior presença de oxigênio em contato com o gel dosímetrico, formando-se nesta região valores maiores de absorbância. Comparando-se os resultados das Fig. $4 a$ e $4 b$, tem-se que na posição vertical para tempos maiores ocorre uma oxidação maior em comparação aos valores da posição horizontal. Na posição horizontal, sem e com tampa, após 15 horas de irradiação, os valores da área sob a curva sigmoidal são maiores em comparação à posição vertical. Este fato pode ser explicado devido ao fato dos íons de $\mathrm{Fe}+3$ na posição vertical devem vencer a barreira gravitacional para se locomoveram, ao contrário da posição horizontal, onde estes íons se 
deslocam e contribuem para valores maiores de absorbância no gel químico. Para intervalos de tempo menores que $5 \mathrm{~h}$, a melhor acomodação das cubetas é para o armazenamento na posição vertical com tampa. Isto se deve principalmente ao valor da área sob a curva sigmoidal em torno de $5 \mathrm{~mm}^{2}$, obtendo-se assim o menor valor de área e consequentemente de oxidação. Neste caso, a proteção por meio da tampa na solução gel é vista como fundamental para que o contato com o oxigênio não tenha contribuições significativas nas leituras (KRON, 1997; MAEYAMA; 2014).

a)

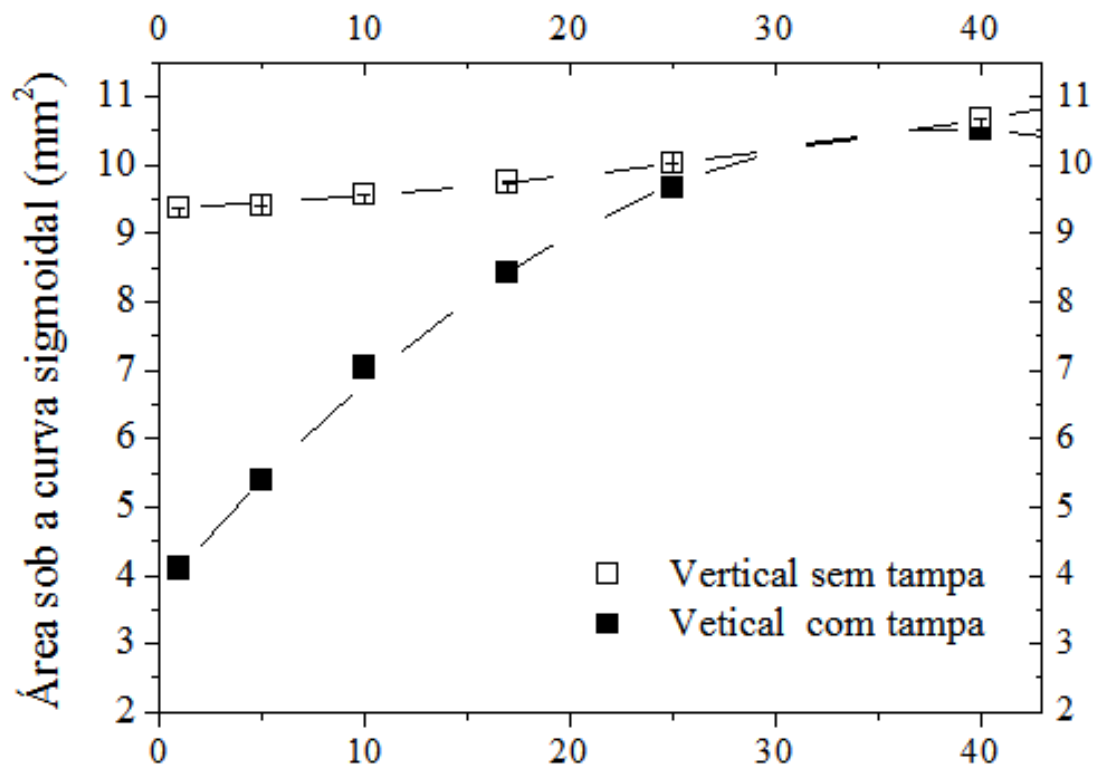

Tempo pós-irradiação (h)

b)

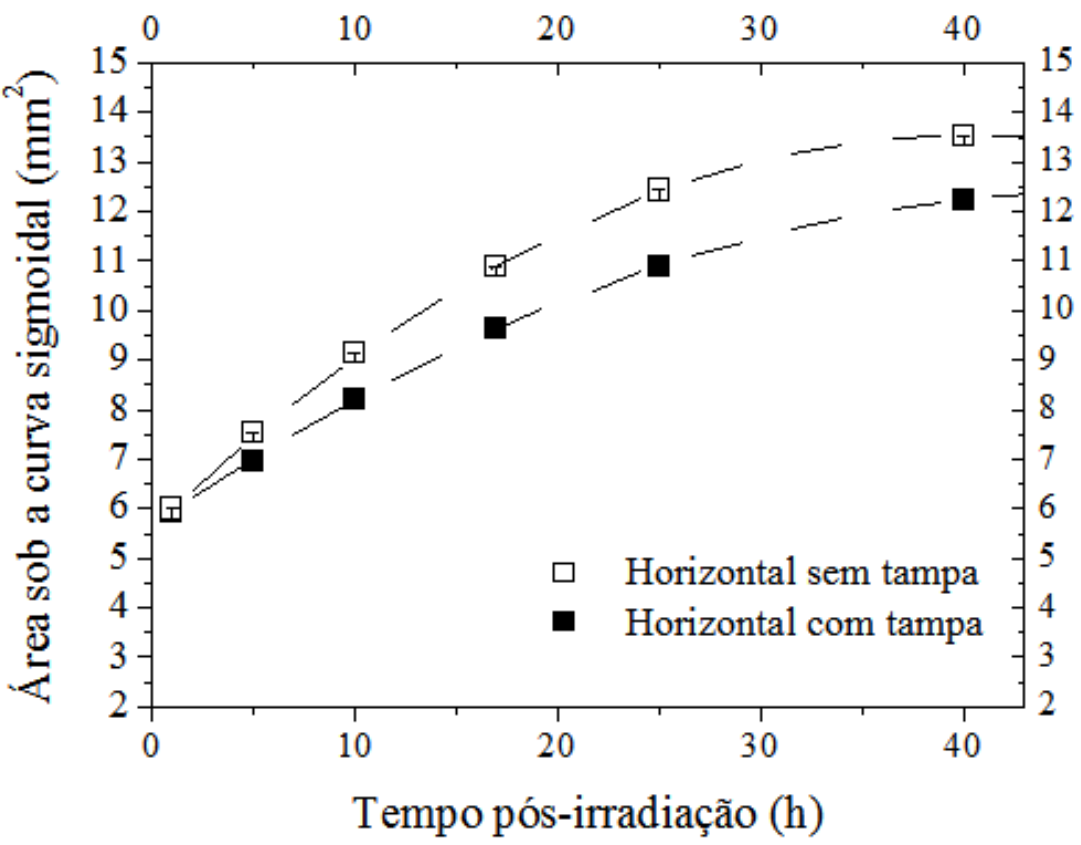

Figura 4. Área sob a curva sigmoidal versus tempo pós-irradiação. a) Cubetas na posição vertical com e sem tampa e b) Cubetas na posição horizontal com e sem tampa. 


\section{CONCLUSÃO}

O método de espectrofotometria proposto apresenta-se como alternativa simples e rápida para determinação da oxidação no dosímetro químico FXG. Os resultados obtidos ao aplicar o método da área de sigmoidal, tendo como referencias cubetas nas posições vertical e horizontal associada na mesma configuração com e sem tampa, apresentaram adequada precisão e sensibilidade para determinação da oxidação. Pode-se concluir dos resultados encontrados: i) a ausência da tampa nas cubetas aumentam consideravelmente os resultados da oxidação, tanto na vertical como na horizontal; ii) valores da oxidação, para tempos acima de 25 horas para cubetas na vertical e após 40 horas nas cubetas na posição horizontal, tendem a serem negligenciados; iii) cubetas dosimétricas na posição horizontal possuem maior oxidação do que na vertical; iv) para tempos menores que 5 horas a melhor acomodação das cubetas é na posição vertical com tampa; v) na posição horizontal os íons não precisam vencer a barreira gravitacional, acarretando em valores maiores de oxidação do que na posição vertical. Desta forma, a pesquisa se torna útil em aplicações da dosimetria química da qual apenas este tipo de gel é capaz de realizar medições em 3D, e a determinação da oxidação relacionada com o tempo é de importância para medições pósirradiação.

\section{AGRADECIMENTOS}

Ao Conselho Nacional de Desenvolvimento Científico - CNPq, sobre o processo n0165466/2015-4 e a FAPEG pelo apoio financeiro. Ao Setor de Radioterapia do Hospital Araújo Jorge (Goiânia-Goiás), por ceder o acelerador linear VARIAN CLINAC 6/100 para as irradiações das cubetas de FXG e ao MCT: Projeto INCT para Metrologia das Radiações em Medicina.

\section{REFERÊNCIAS BIBLIOGRÁFICAS}

1. ALVA-SÁNCHEZ, M.S., OLIVEIRA, L.N., PETCHEVIST, P.C., MOREIRA, M.V., ALMEIDA, A., (2014). Beta planar source quality assurance with the Fricke xylenol gel dosimeter. Radiat. Phys. Chem. 96, 56-59.

2. ATTIX, F.H. (1993). Introduction to Radiological Physics and Radiation Dosimetry, John Wiley \& Sons.

3. BERO, M.A., GILBOY, W.B., GLOVER, P.M., KEDDIE, J.L., (1999). Three-dimensional radiation dose measurements with Ferrous Benzoic Acid Xylenol Orange in Gelatin gel and optical absorption tomography. Nucl. Instr. Meth. Phys. B, 422, 617-620.

4. BERO, M.A., GILBOY, W.B., GLOVER, P.M., EL-MASRI, H. M., (2000). Tissue-equivalent gel for non-invasive spatial radiation dose measurements. Nucl. Instr. Meth. Phys. B, 166, 820-825.

5. BERO, M.A., GILBOY, W.B., GLOVER, P.M., (2001). Radiochromic gel dosemeter for threedimensional dosimetry. Radiat. Phys. Chem. 61, 433-435.

6. CALCINA, C.S.G., OLIVEIRA, L.N., ALMEIDA, C.E., ALMEIDA, A., (2007). Dosimetric parameters for small field sizes using Fricke xylenol gel, thermoluminescent, film dosimeters, and an ionization chamber. Phys. Med. Biol. 52, 1431-1439.

7. CALDEIRA, A.M., NETO, A.M., BENTO, A.C., BAESSO, M.L., SILVA, M.A., DE ALMEIDA A. (2007a). Behavior of oxidation in the radiochromic gel dosimeter through photoacoustic 
technique measurements. Appl Radiat Isot. 2007, 65, 605-609.

8. CALDEIRA, A.M.F., DE ALMEIDA, A., NETO, A.M., BAESSO, M., BENTO, A.C., SILVA, M.A., (2007b). Fricke Xylenol Gel characterization using a photoacustic technique. Nucl. Instr. Meth. Phys. A, 582, 484-488.

9. DAVIES, J.B., BALDOCK, C., (2008). Sensitivity and stability of the Fricke-gelatin-xylenol orange gel dosimeter. Radiat. Phys. Chem. 77, 690-696.

10. DAVIES, J.B., BALDOCK, C., (2010). Temperature dependence on the dose response of the Fricke-gelatin-xylenol orange gel dosimeter. Radiat. Phys. Chem. 79, 660-662.

11. DAVIES, J.B., BOSI, S.G., BALDOCK., C., (2013). Dosimetry aspects of a non-diffusing genipingelatin gel. Radiat. Phys. Chem. 83, 19-27.

12. JOHNS, H.E., CUNNINGHAM, J.R. (1983). The Physics of Radiology, 4th ed. American Lecture, Ilinois: Charles Thomas.

13. KHAN, F.M. The physics of radiation therapy.(1993) 3rd ed., Philadelphia: Lippincott Williams \& Wilkins, 1993.

14. KRON, T.D., JONAS, D., POPE, J.M., (1997). Fast T1 imaging of dual gel samples for diffusion measurements in NMR dosimetry gels. Magn. Reson. Imaging. 15, 211-221.

15. MAEYAMA, T., FUKUNISHI, N., ISHIKAWA, K.L., FURUTA, T., FUKASAKU, K., TAKAGI, S., NODA, S., HIMENO, R., FUKUDA S., (2014). A diffusion-free and linear-energy-transfer-independent nanocomposite Fricke gel dosimeter. Radiat. Phys. Chem. 96, 92-96.

16. OliVEIRA, L.N., CALCINA, C.S.G., PARADA, M.A., ALMEIDA, C.E., ALMEIDA, A., (2007). Ferrous Xylenol Gel Measurements for 6 and 10 MV Photons in Small Fiel Sizes. Braz. J. Phys. 27 11411146.

17. OLIVEIRA, L.N., ZIMMERMAN, R.L, MOREIRA, M.V., ILA, D., ALMEIDA, A., (2009a). Determination of diffusion coefficient in Fricke Xylenol gel dosimeter after electron beam bombardment. Surf. Coating. Tech. 203, 2367-2369.

18. OliVEIRA, L.N., CALCINA, C.S.G., PARADA, M.A., ALMEIDA, C.E., ALMEIDA, A., (2009b). 6 MV Wedge Photon Beam Profiles with the Fricke Xylenol Gel Dosimeter. Braz. J. Phys. 39, 615-618.

19. PIRANI, L.F., MOREIRA, M.V., COSTA, J.J.L, OLIVEIRA, L.N., CALDAS, L.V.E, ALMEIDA, A., (2013). Fricke dosimeter gel measurements of the profiles of shielded fields. Appl. Radiat. Isot. 82, 239-241.

20. PIRANI, L.F., OLIVEIRA, L.N., PETCHEVIST, P.C.D., MOREIRA, M.V., ILA, D., ALMEIDA, A., (2009). New chemical Fricke gel radiation dosimeter. J. Radioanal. Nucl. Chem. 280, 259-262.

21. SAMPAIO, F.G.A., OLIVEIRA, L.N., MOREIRA, M.V., PETCHEVIST, P.C.D., ALMEIDA, C.E., ALMEIDA, A., (2013a). 8 and 10 MeV Electron Beams Small Field-Size Dosimetric Parameters Through the Fricke Xylenol Gel Dosimeter. IEEE Trans. Nucl. Sci. 60, 572-577.

22. SAMPAIO, F.G.A., DEL LAMA, L.S., SATO, R., DE OLIVEIRA, D.M.M., CZELUSNIAK, C., OLIVEIRA, L.N., ALMEIDA, A., (2013b). Quality Assurance of a Two-Dimensional CCD Detector System Applied in Dosimetry. IEEE Trans. Nucl. Sci. 60, 810-816. 